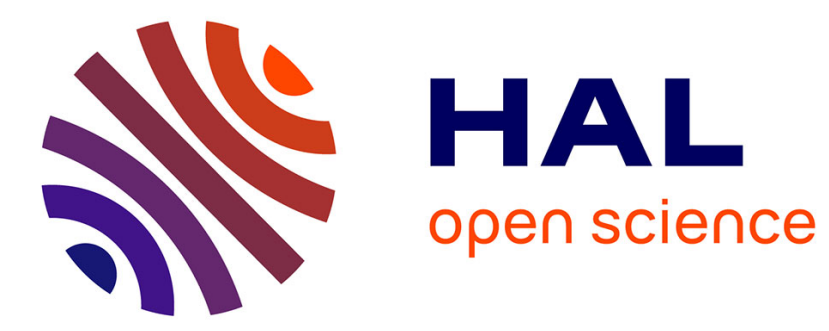

\title{
Coexistence of schwannomatosis and glioblastoma in two families
}

\author{
C. Deiller, Julien Van-Gils, C. Zordan, J. Tinat, H. Loiseau, T. Fabre, Claire \\ Delleci, J. Cohen, M. Vidaud, B. Parfait, et al.
}

\section{- To cite this version:}

C. Deiller, Julien Van-Gils, C. Zordan, J. Tinat, H. Loiseau, et al.. Coexistence of schwannomatosis and glioblastoma in two families. European Journal of Medical Genetics, 2019, 62 (8), pp.103680. 10.1016/j.ejmg.2019.103680 . hal-03209350

\section{HAL Id: hal-03209350 \\ https://hal.science/hal-03209350}

Submitted on 25 Oct 2021

HAL is a multi-disciplinary open access archive for the deposit and dissemination of scientific research documents, whether they are published or not. The documents may come from teaching and research institutions in France or abroad, or from public or private research centers.
L'archive ouverte pluridisciplinaire HAL, est destinée au dépôt et à la diffusion de documents scientifiques de niveau recherche, publiés ou non, émanant des établissements d'enseignement et de recherche français ou étrangers, des laboratoires publics ou privés.

\section{다)(1) $(5$}

Distributed under a Creative Commons Attribution - NonCommerciall 4.0 International 


\section{Coexistence of schwannomatosis and glioblastoma in two families}

Caroline Deiller $r^{1, a}$, Julien Van-Gils ${ }^{1,2, *, a}$, Cécile Zordan ${ }^{1}$, Julie Tinat ${ }^{1}$, Hugues Loiseau ${ }^{3}$, Thierry Fabre ${ }^{4}$, Claire Delleci ${ }^{5}$, Joëlle Cohen ${ }^{6}$, Michel Vidaud ${ }^{6,7}$, Béatrice Parfait6,7, Cyril Goizet $^{1,2}$.

${ }^{1} \mathrm{CHU}$ Bordeaux, Service de Génétique Médicale, Bordeaux, France

2Laboratoire MRGM, INSERM U1211, Univ. Bordeaux, Bordeaux, France

${ }^{3} \mathrm{CHU}$ Bordeaux, Service de neurochirurgie, Bordeaux, France

${ }^{4} \mathrm{CHU}$ Bordeaux, Service d'orthopédie, Bordeaux, France

${ }^{5} \mathrm{CHU}$ Bordeaux, Service de Médecine Physique et Réadaptation, Bordeaux, France

${ }^{6}$ Service de Génétique et Biologie Moléculaires, Hôpital Cochin, Assistance PubliqueHôpitaux de Paris, Paris, France

7UMR INSERM 1016, Institut Cochin, Université Paris Descartes, Sorbonne Paris Cité, Paris, France

${ }^{a}$ Both authors contributed equally to this work.

${ }^{*}$ Corresponding Author:

Julien Van-Gils

$\mathrm{CHU}$ Bordeaux

Place Amélie Raba-Léon

33076 Bordeaux cedex

Phone +5557820353

julien.van-gils@chu-bordeaux.fr 


\section{Abstract}

Schwannomatosis is a rare affection predisposing to multiple peripheral neurologic tumors development. Approximatively, one third of patients with schwannomatosis are carriers of a germline mutation in LZTR1 (Leucin Zipper Transcription Regulator 1). Tumorigenesis in schwannomatosis responds to a somatic 5-hit/3-step mechanism resulting in a loss of function (LOF) of LZTR1 and the contiguous genes of locus 22q11.2q12.2. Effectively, LZTR1 is mapped on 22q11.2 and centromeric to SMARCB1 also implicated in the determinism of schwannomatosis and NF2, responsible for neurofibromatosis type 2. On a somatic point of view, LZTR1 mutations are known to drive with a significant frequency glioblastoma (GB) development. We report here two families in which segregate both multiple schwannomas and GB. In the first family, the proband received a diagnosis with of schwannomatosis after a surgery for a lumbar schwannoma at age 43, molecularly confirmed by identification of a germline heterozygous mutation in LZTR1. Her father, having unremarkable medical history deceased from an apparently isolated GB at age 59 . In the second family, LZTR1-related schwannomatosis was diagnosed in the index case at age 70 after multiple schwannomas surgeries. Her elder sister had no neurological medical history before occurrence of a lethal GB at age 78. Molecular analysis of GB sample from both affected relatives showed the presence of the familial mutation. These observations hypothesize a potential link between schwannomatosis and the GB development.

Keywords: Schwannomatosis, Glioblastoma, Neurofibromatosis, LZTR1, SMARCB. 


\section{Introduction}

Schwannomatosis (OMIM \#162091) is a rare predisposition to develop multiple peripheral schwannomas. The diagnosis is based on clinical examination and molecular analysis (Table 1). Although mutations in SMARCB1 were first described ${ }^{1}$, loss of function (LOF) mutations in LZTR1 were recently incriminated ${ }^{2,3}$. Germline SMARCB1 mutations account for $48 \%$ of familial and $9.8 \%$ of sporadic cases and germline LZTR1 mutations are found in approximately $38 \%$ of familial and $22-30 \%$ of sporadic SMARCB1-negative cases ${ }^{4}$. Tumorigenesis in schwannomatosis responds to a somatic 5-hit/3-step mechanism resulting in a loss of function (LOF) of LZTR1 and of the contiguous genes of locus 22q11.2q12.2, SMARCB1 and NF2, implicated in neurofibromatosis type 2.

Moreover, somatic mutations and biallelic inactivation of LZTR1 are identified in $22 \%$ of sporadic GB, LZTR1 loss driving immortalization and proliferation of cells, and acting thus as a classical tumor suppressor gene ${ }^{5}$.

We describe two non-related families in which germline LZTR1 mutations were found in a proband displaying schwannomatosis. In each family, the LZTR1 mutations was also identified at a somatic level in an isolated glioblastoma of a relative.

\section{Material and methods}

\section{Patients and samples}

Blood samples were obtained from index cases after accurate information and signed consent in accordance with law of bioethics $n^{\circ} 2004-800$ of 06.08 .2004 , revised 7 . July 2011. DNA was isolated from peripheral blood leucocytes using standard procedures. Genetic analysis of tumor samples was assessed after information and consent of living 
relatives. DNA extraction of GB samples conserved in paraffin block (FFPE, formalin-fixed, paraffin-embedded tissue) was performed with the Maxwell囚 16 FFPE Tissue LEV DNA Kit.

\section{Targeted NGS (Next Generation Sequencing) genotyping of NF2, SMARCB1 and LZTR1}

Genotyping experiments were performed on the NGS facility of the Cochin hospital, Paris (Assistance Publique - Hôpitaux de Paris, France). A custom Ampliseq panel targeting NF2, SMARCB1 and LZTR1 was developed (reference IAD51599_119, ThermoFisherScientific). Molecular diagnosis was performed using a targeted Next-Generation Sequencing method genotyping NF2, SMARCB1 and LZTR1 in blood cells of the probands and GB tissue of their relative in two unrelated families. NGS libraries preparation followed by amplification and purification, emulsion PCR, enrichment, loading on Ion 318 an Ion Personal Genome Machine ${ }^{\circledR}\left(\mathrm{PGM}^{\mathrm{T} M}\right)$ System (Life Technologies), data collection and sequence alignment were performed as already described ${ }^{6,7}$.

Loss Of Heterozygosity (LOH) study at SNP (Single Nucleotide Polymorphism) level.

Analysis of informative SNPs allelic ratio was performed at NF2, SMARCB1, and LZTR1 locus. LOH was excluded for informative SNPs with allelic ratio ranging from 40 to $60 \%$.

\section{Observations}

\section{Family 1}

The proband was a 43-year-old woman referred to our genetic department after surgery of a lumbar schwannoma. She suffered from atypical left sciatica for several years. Medullar 
MRI showed a schwannoma at L2 level. Her personal history was marked by removal of a schwannoma in her left arm at age 18. Her father (Figure 1), after uneventful medical history, presented at age 59 a mild progressive right hemiparesia associated with one right facial motor partial seizure that led to discover a left rolandic GB multiforme. He died 6 months later. Further family history was not contributory as no other relative was symptomatic or underwent MRI-imaging investigating central nervous system and vestibular nerves. Analysis of NF2 and SMARCB1 was negative in the blood of the proband and in the GB. A germline heterozygous variant in exon 10 of LZTR1 was identified in the blood of the proband, c.1126C>T (NM_006767.3) leading to a predicted truncated protein p.(GIn367Ter). This mutation was present in her father's GB with an allelic ratio of 0.7 (c.1126C>T versus wild type) consistent with a loss of heterozygosity in the FFPE GB in the context of a cellular heterogeneity. DNA fragmentation is usually observed with FFPE tissue preparation $^{6}$; this did not allow genes copy number analysis in the FFPE GB. Nevertheless, the $\mathrm{LOH}$ was confirmed by unbalanced allelic ratio of 0.7 for two $L Z T R 1$, one SMARCB1 and one NF2 intragenic SNPs suggesting that LOH is extended beyond LZTR1 locus.

\section{Family 2}

The proband was a 70-year-old woman with schwannomatosis diagnosed after multiple schwannoma surgeries (three in the left leg, one in the left arm and two on the cauda equine) and episodes of left sciatica. Medullar MRI showed diffuse schwannomas in the cauda equine. In the family history, her elder sister (Figure 1), without any particular antecedent, has been hospitalized at age 77 for a left hemiparesia evolving since a month. She was diagnosed with right fronto-parietal GB and died 18 months later. There were no cases of neuropathic anomalies in relatives and MRI imaging was not performed to assess CNS status. Study of NF2 and SMARCB1 was negative in blood DNA of the proband, while analysis of $L Z T R 1$ revealed a previously described heterozygous germline mutation in exon 
2, c.264-13G>A leading to a predicted truncated protein p.(Lys89CysfsTer16) $)^{2}$. This mutation was identified at the heterozygous state in her sister's GB. The balanced allelic ratio observed for one LZTR1 and two NF2 informative SNPs led us to conclude in the absence of $\mathrm{LOH}$.

Both LZTR1 variants identified in this study have been deposited in the Leiden Open Variation Databases (https://databases.lovd.nl/shared/genes/LZTR1).

\section{Discussion}

LZTR1 is a tumor suppressor gene. LZTR1 is highly conserved throughout evolution and contains two functional domains with a kelch-BTB-BACK-BTB-BACK motive. The BTB domains of the protein interact with the cullin 3 (CUL3)-RING ubiquitin ligase (CRL3) complex, a multi-subunit RING-class E3 ligase involved in protein mono- and polyubiquitination $^{5,8}$. It has been recently shown that LZTR1 binds substrates including RAS via the tandemly repeated Kelch domains allowing RAS ubiquitination by CRL3 complex ${ }^{9,10}$. LZTR1 loss abrogates RAS ubiquitination by disrupting the formation of the RAS-LZTR1CUL3 complex, leads to increased RAS activity and phosphorylation of MEK/ERK proteins and increases growth rate of different cellular models including Schwann cells. One of its partners is CULLIN3 implicated in mitosis division. Frattini et al. (2013) observed that LZTR1 loss of function mutations in GB cause altered interaction between CULLIN3 and LZTR1 leading to cell proliferation and that LZTR1 inactivation drives the self-renewal and growth of glioma spheres model 5 .

LZTR1 mutations are found in schwannomas and GB. Both tumors have a similar genesis pattern: the combination of point mutation and LOH in LZTR1 locus. 
Sporadic GB genesis follows a classical 2-hit model for a tumor suppressor gene. In schwannomas, a 5-hit/3-step model was suggested for tumorigenesis where the germline LZTR1 mutation is associated to the (partial) loss of the other copy of chromosome 22 including LZTR1, SMARCB1 and NF2 and somatically acquired tumor-specific mutation in NF2. ${ }^{4}$. In our study, the locus $22 q 11.21 q 12.2$ showed LOH in tumoral DNA in GB of family 1 suggesting that the genesis of the tumor could be linked to additional hits including SMARCB1 and NF2 following at least in part the model of schwannomas. The contribution of these additional hits as driver or only passenger in the tumorigenesis of GB remains to be clarified. In family 2, LOH is absent in GB DNA. Biallelic LOF of LZTR1 secondary to single or multiple exons deletion or duplication can be involved but FFPE sample was not suitable for the identification of these possible events and frozen GB was not available. Therefore, we cannot exclude a second hit in LZTR1. Moreover, multiple other genes are known to drive GB tumorigenesis ${ }^{5}$. A second hit in another GB related gene may alternatively explain GB development in family 2.

Clinical features of LZTR1 mutated patients with schwannomatosis and their relatives have been described before $2,3,11$. No co-occurrence of GB has been reported so far (62 index cases and 15 relatives). However recently, mutations in LZTR1 have also been involved in a small proportion of patients with Noonan syndrome, a rare neurodevelopmental syndrome $^{12}$. A recurrent mixed glioma tumor of oligoastrocytoma type was described in such a patient ${ }^{13}$. Our observations raise the question of a link between LZTR1-related schwannomatosis and GB development, as the familial mutation may be here the first hit in the GB. Recently, LZTR1 mutations leading to GB were observed mainly in the functional domain of the protein and were all expected to produce a LOF ${ }^{5}$. The point mutations described in sporadic GB have not been identified in patients with schwannomatosis. 
Schwannomatosis-related mutations are distributed all along LZTR1 and expected to lead to a LOF. In our observation, the same LZTR1 mutations were identified in both conditions.

Similarly, SMARCB1 mutations are associated with schwannomatosis and rhabdoid tumors (RT). Vitte et al. (2017) generated different tissue and developmental stage-specific conditional knockout mice carrying Smarcb1 and/or Nf2 deletion and identified the mechanisms that differ between schwannomas and RT genesis: biallelic loss of Smarcb1 in neural crest cells during early embryonic development is associated with postnatal RT formation, Nf2 loci being functional. Their model also displayed that biallelic loss of Nf2 is mandatory for schwannoma tumorigenesis whereas loss of Smarcb1 is not ${ }^{14}$. In light of these observations, we can hypothesize that the schwannomatosis-related genes might participate in malignant tumors development.

In conclusion, two rare conditions segregate with LZTR1 mutations in our unrelated families. Recent articles highlight the key role of LZTR1 mutations and the similar mechanism implicated in both pathologies. Nevertheless, we need further observations to ascertain whether GB can be included in the tumor spectrum of schwannomatosis.

\section{Acknowledgments}

We thank the patients and their families.

We thank the Biological Resources Center, Tumorothèque, Hôpital Cochin, Paris, France for DNA extraction from FFPE samples. 
Table 1: Diagnostic criterias for schwannomatosis adapted from Kehrer-Sawatzki et al (2017).

\begin{tabular}{|c|}
\hline Combined molecular and clinical diagnosis \\
\hline 22 tumors with 22q LOH and 2 different somatic NF2 mutations \\
AND \\
$\geq 2$ pathologically confirmed schwannomas or meningiomas \\
\hline OR \\
\hline Germline SMARCB1 or LZTR1 pathogenic mutation \\
AND \\
1 pathologically confirmed schwannoma or meningioma \\
\hline
\end{tabular}

\section{Clinical diagnosis}

$\geq 2$ non-intradermal schwannomas, 1 pathologically confirmed and no bilateral vestibular schwannoma by high quality MRI ( some mosaic NF2 patients will be included in this diagnosis at a young age and some schwannomatosis patient may have unilateral vestibular schwannoma or multiple meningiomas)

\section{OR}

1 pathologically confirmed schwannoma or intracranial meningioma AND an affected first-degree relative.

Exclusion criteria for schwannomatosis :

- Germline pathogenic NF2 mutation

- Diagnostic criteria for NF2 fulfilled

- First-degree relative with NF2

- Schwannomas occur exclusively in region of previous radiation therapy 


\section{FIGURE}

(A) Family 1:

Mutation in exon 10 of LZTR1

c. $1126 \mathrm{C}>\mathrm{T}, \mathrm{p} .\left(\mathrm{G} \ln 367^{*}\right)$

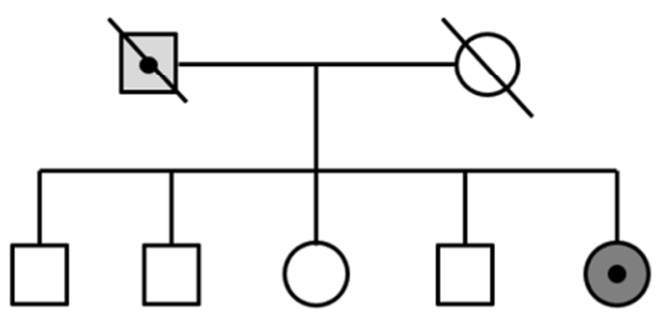

(B) Family 2 :

Mutation in intron 2 of LZTR1

c. 264-13G>A, p.Lys89Cysfs*16

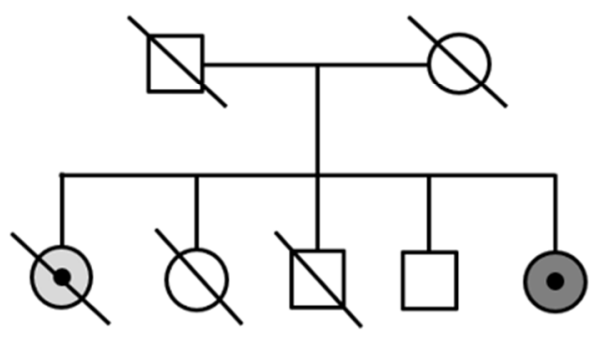

Figure 1

Pedigree charts

Individuals diagnosed with schwannomatosis are indicated as affected (dark gray), individuals with glioblastoma are indicated in light gray. Patients marked with a black dot are heterozygous for the familial mutation. (A) Pedigree of family 1. (B) Pedigree of family 2. 


\section{REFERENCES}

1. Hulsebos TJM, Plomp AS, Wolterman RA, et al. Germline Mutation of INI1/SMARCB1 in Familial Schwannomatosis. Am J Hum Genet 2007;80(4):805-810.

2. Piotrowski A, Xie J, Liu Y F., et al. Germline Loss-of-Function Mutations in LZTR1 Predispose to an Inherited Disorder of Multiple Schwannomas. Nat Genet 2014;46:182-187.

3. Paganini I, Chang V Y, Capone G L, et al. Expanding the Mutational Spectrum of LZTR1 in Schwannomatosis. Eur J Hum Genet 2015;23:963-968.

4. Kehrer-Sawatzki H, Farschtschi S, Mautner V-F, and Cooper D N. The Molecular Pathogenesis of Schwannomatosis, a Paradigm for the Co-Involvement of Multiple Tumour Suppressor Genes in Tumorigenesis. Hum Genet 2017;136:129-148.

5. Frattini V, Trifonov V, Minhow Chan J, et al. The Integrated Landscape of Driver Genomic Alterations in Glioblastoma. Nat Genet 2013;45:1141-1149.

6. Pasmant E, Louvrier C, Luscan A, et al. Neurofibromatosis type 2 French cohort analysis using a comprehensive NF2 molecular diagnostic strategy. Neurochirurgie 2015 Jun 11. pii: S0028-3770(15)00082-X.

7. Louvrier C, Pasmant E, Briand-Suleau A, et al. Targeted next-generation sequencing for differential diagnosis of neurofibromatosis type 2, schwannomatosis, and meningiomatosis. Neuro Oncol. 2018 Jun 18;20(7):917-929.

8. Zimmerman ES, Schulman BA, Zheng N. Structural assembly of cullin-RING ubiquitin ligase complexes. Curr Opin Struct Biol. 2010 Dec;20(6):714-21.

9. Steklov M, Pandolfi S, Baietti MF, et al. Mutations in LZTR1 drive human disease by dysregulating RAS ubiquitination. Science. 2018 Dec 7;362(6419):1177-1182.

10. Bigenzahn JW, Collu GM, Kartnig F, et al. LZTR1 is a regulator of RAS ubiquitination and signaling. Science. 2018 Dec 7;362(6419):1171-1177.

11. Hutter S, Piro RM, Reuss DE, et al. Whole exome sequencing reveals that the majority of schwannomatosis cases remain unexplained after excluding SMARCB1 and LZTR1 germline variants. Acta Neuropathol 2014;128(3):449-452.

12. Yamamoto GL, Aguena M, Gos M, et al. Rare variants in SOS2 and LZTR1 are associated with Noonan syndrome. J Med Genet. 2015 Jun;52(6):413-21.

13. Jacquinet A, Bonnard A, Capri $Y$, et al. Oligo-astrocytoma in LZTR1-related Noonan syndrome. Eur J Med Genet. 2019 Jan 19. pii: S1769-7212(18)30629-3. 
14. Vitte J, Gao F, Coppola G, Judkins A R., Giovannini M, Timing of Smarcb1 and Nf2 inactivation determines schwannoma versus rhabdoid tumor development. Nat Commun 2017;8(1):300. 